\title{
Editorial
}

\section{Artificial Intelligence and Its Applications 2014}

\author{
Yudong Zhang, ${ }^{1}$ Saeed Balochian, ${ }^{2}$ Praveen Agarwal, ${ }^{3}$ Vishal Bhatnagar, \\ and Orwa Jaber Housheya ${ }^{5}$ \\ ${ }^{1}$ School of Computer Science and Technology, Nanjing Normal University, Nanjing, Jiangsu 210023, China \\ ${ }^{2}$ Department of Electrical Engineering, Islamic Azad University, Gonabad Branch, Gonabad 96916-29, Iran \\ ${ }^{3}$ Department of Mathematics, Anand International College of Engineering, Jaipur 303012, India \\ ${ }^{4}$ Ambedkar Institute of Advanced Communication Technologies and Research, Government of NCT of Delhi, \\ Geeta Colony, Delhi 110031, India \\ ${ }^{5}$ Department of Chemistry, Arab American University, Jenin, State of Palestine
}

Correspondence should be addressed to Yudong Zhang; zhangyudong@njnu.edu.cn

Received 22 February 2016; Accepted 23 February 2016

Copyright (c) 2016 Yudong Zhang et al. This is an open access article distributed under the Creative Commons Attribution License, which permits unrestricted use, distribution, and reproduction in any medium, provided the original work is properly cited.

Artificial intelligence (AI) researches the intelligence exhibited by machines. It creates revolutionized information technology. The world famous companies like Google, Yahoo, Facebook, Baidu, and so forth have spent millions of dollars to research on developing new algorithms on AI. Nevertheless, there are a number of challenging issues in realistic applications due to fast-growing large and complex problems.

This special issue aims to bring together academia and industry experts to report on the recent developments on artificial intelligence and its applications, in every aspect of artificial intelligence technology, including machine learning, data mining, computer vision, multiagent systems, evolutionary computation, deep learning, and fuzzy logic. The primary guideline was to either demonstrate the most significant developments on the topics of AI or apply AI-related algorithms in real-life scenarios.

In the paper entitled "A Comprehensive Survey on Particle Swarm Optimization Algorithm and Its Applications," Y. Zhang et al. investigated the particle swarm optimization (PSO). Their survey presented a comprehensive investigation of PSO. On one hand, they provided advances with PSO, including its modifications (including quantumbehaved PSO, bare-bones PSO, chaotic PSO, and fuzzy PSO), population topology (as fully connected, von Neumann, ring, star, random, etc.), hybridization (with genetic algorithm, simulated annealing, Tabu search, artificial immune system, ant colony algorithm, artificial bee colony, differential evolution, harmonic search, and biogeography-based optimization), extensions (to multiobjective, constrained, discrete, and binary optimization), theoretical analysis (parameter selection and tuning and convergence analysis), and parallel implementation (in multicore, multiprocessor, GPU, and cloud computing forms). On the other hand, they offered a survey on applications of PSO to the following eight fields: electrical and electronic engineering, automation control systems, communication theory, operations research, mechanical engineering, fuel and energy, medicine, chemistry, and biology. It is hoped that this survey would be beneficial for the researchers studying PSO algorithms.

In the paper entitled "Model of Multilayer Knowledge Diffusion for Competence Development in an Organization," P. Różewski and J. Jankowski proposed several models with the main goal of simulating diffusion and explaining the nature of knowledge diffusion. They extended existing approaches by using multilayer diffusion model and focused on analysis of competence development process. The proposed model described competence development process in a new way through horizontal and vertical knowledge diffusion in multilayer network. In the network, agents collaborated and interchanged various kinds of knowledge through different layers and these mutual activities affected the competencies in a positive or negative way. Taking into consideration worker's cognitive and social abilities and the previous level of competence, the new competence level 
can be estimated. Their model was developed to support competence management in different organizations.

In the paper entitled "Landslide Occurrence Prediction Using Trainable Cascade Forward Network and Multilayer Perceptron," M. S. Al-batah et al. introduced two models of artificial neural network, namely, multilayer perceptron (MLP) and cascade forward neural network (CFNN), to predict the landslide hazard map of Penang Island. These two models were tested and compared using eleven machinelearning algorithms: Levenberg Marquardt, Broyden Fletcher Goldfarb, resilient backpropagation, scaled conjugate gradient, conjugate gradient with Beale, conjugate gradient with Fletcher Reeves updates, conjugate gradient with Polakribiere updates, one step secant, gradient descent, gradient descent with momentum and adaptive learning rate, and gradient descent with momentum algorithm. The performance of the landslide prediction depends on the input factors beside the prediction method. In this research work, 14 input factors were used. The prediction accuracies of networks were verified using the area under curve for the receiver operating characteristics. The results indicated that the best prediction accuracy was achieved using the CFNN network with the Levenberg Marquardt learning algorithm, that is, $82.89 \%$ for the training data set and $81.62 \%$ for the testing data set.

In the paper entitled "Optimization of Train Trip Package Operation Scheme," L. Tong et al. firstly analyzed the related factors of train trip package transportation from its organizational forms and characteristics. Then an optimization model for train trip package transportation was established to provide optimum operation schemes. The proposed model was solved by the genetic algorithm. At last, the paper tested the model on the basis of the data of 8 regions. The results showed that the proposed method is feasible for solving operation scheme issues of train trip package.

In the paper entitled "A Self-Adaptive Hidden Markov Model for Emotion Classification in Chinese Microblogs," L. Liu et al. proposed a modified version of hidden Markov model (HMM) classifier, called self-adaptive HMM, the parameters of which were optimized by particle swarm optimization algorithms. Since manually labeling large-scale dataset was difficult, Liu et al. also employed the entropy to decide whether a new unlabeled tweet will be contained in the training dataset after being assigned an emotion using the proposed HMM-based approach. In the experiment, about 200,000 Chinese tweets from Sina Weibo were collected. The results showed that the $F$-score of the approach gets $76 \%$ on happiness and fear and 65\% on anger, surprise, and sadness. In addition, the self-adaptive HMM classifier outperformed Naive Bayes and support vector machine on recognition of happiness, anger, and sadness.

In the paper entitled "Automatic Classification of Remote Sensing Images Using Multiple Classifier Systems,” B. Yang et al. aimed to correctly identify land use types reflected in remote sensing images. Support vector machine, maximum likelihood classifier, backpropagation neural network, fuzzy $c$-means, and minimum distance classifier were combined to construct three multiple classifier systems (MCSs). Two MCSs were implemented, namely, comparative major voting
(CMV) and Bayesian average (BA). One method called WAAHP was proposed, which introduced analytic hierarchy process into MCS. Classification results of base classifiers and MCSs were compared with the ground-truth map. Accuracy indicators were computed and receiver operating characteristic curves were illustrated, so as to evaluate the performance of MCSs. Experimental results showed that employing MCSs can increase classification accuracy significantly, compared with base classifiers. From the accuracy evaluation result and visual check, the best MCS is WA-AHP with overall accuracy of $94.2 \%$, which overmatches BA and rivals CMV. The producer's accuracy of each land use type proves the good performance of WA-AHP. Therefore, MCS is superior to base classifiers in remote sensing image classification, and WAAHP is an efficient MCS.

In the paper entitled "A Novel Tournament Selection Based Differential Evolution Variant for Continuous Optimization Problems," Q. Abbas et al. proposed a novel tournament based parent selection variant of differential evolution (DE) algorithm. The proposed variant enhanced searching capability and improved convergence speed of $\mathrm{DE}$ algorithm. This paper also presented a novel statistical comparison of existing DE mutation variants, which categorized these variants in terms of their overall performance. Experimental results showed that the proposed DE variant had significance performance over other DE mutation variants.

In the paper entitled "Manifold Learning with SelfOrganizing Mapping for Feature Extraction of Nonlinear Faults in Rotating Machinery," L. Liang et al. proposed a new method for extracting the low-dimensional feature automatically with self-organization mapping manifold for the detection of rotating mechanical nonlinear faults (such as rubbing and pedestal looseness). Under the phase space reconstructed by single vibration signal, the self-organization mapping (SOM) with expectation maximization iteration algorithm was used to divide the local neighborhoods adaptively without manual intervention. After that, the local tangent-space alignment algorithm was adopted to compress the high-dimensional phase space into low-dimensional feature space. The proposed method takes advantages of the manifold learning in low-dimensional feature extraction and adaptive neighborhood construction of SOM and can extract intrinsic fault features of interest in two-dimensional projection space. To evaluate the performance of the proposed method, the Lorenz system was simulated and rotation machinery with nonlinear faults was obtained for test purposes. Compared with the holospectrum approaches, the results reveal that the proposed method is superior in identifying faults and effective for rotating machinery condition monitoring.

In the paper entitled "Modifying Regeneration Mutation and Hybridising Clonal Selection for Evolutionary Algorithms Based Timetabling Tool," T. Thepphakorn et al. outlined the development of a new evolutionary algorithms based timetabling (EAT) tool for solving course scheduling problems that include a genetic algorithm (GA) and a memetic algorithm (MA). Reproduction processes may generate infeasible solutions. Previous research used 
repair processes that were applied after a population of chromosomes was generated. This research developed a new approach which (i) modified the genetic operators to prevent the creation of infeasible solutions before chromosomes were added to the population and (ii) included the clonal selection algorithm (CSA) and the elitist strategy (ES) to improve the quality of the solutions produced. This approach was adopted by both the GA and MA within the EAT. The MA was further modified to include hill climbing local search. The EAT program was tested using 14 benchmark timetabling problems from the literature using a sequential experimental design, which included a fractional factorial screening experiment. Experiments were conducted to (i) test the performance of the proposed modified algorithms, (ii) identify which factors and interactions were statistically significant, (iii) identify appropriate parameters for the GA and MA, and (iv) compare the performances of the various hybrid algorithms. The genetic algorithm with modified genetic operators produced an average improvement of over $50 \%$.

In the paper entitled "Fuzzy Wavelet Neural Network Using a Correntropy Criterion for Nonlinear System Identification," L. L. S. Linhares et al. investigated the fuzzy wavelet neural networks (FWNNs) that are an efficient tool to identify nonlinear systems. In these structures, features related to fuzzy logic, wavelet functions, and neural networks are combined in an architecture similar to the adaptive neurofuzzy inference systems (ANFIS). In practical applications, the experimental data set used in the identification task often contains unknown noise and outliers, which decrease the FWNN model reliability. In order to reduce the negative effects of these erroneous measurements, their work proposed the direct use of a similarity measure based on information theory in the FWNN learning procedure. The mean squared error (MSE) cost function was replaced by the maximum correntropy criterion (MCC) in the traditional error backpropagation (BP) algorithm. The input-output maps of a real nonlinear system studied were identified from an experimental data set corrupted by different outliers rates and additive white Gaussian noise. The results demonstrated the advantages of the proposed cost function using the MCC as compared to the MSE. This work also investigated the influence of the kernel size on the performance of the MCC in the BP algorithm, since it is the only free parameter of correntropy.

In the paper entitled "A Novel WLAN Client Puzzle against DoS Attack Based on Pattern Matching," A. Ordi et al. analyzed the popularity of 802.11 based networks and pointed out that they suffered several types of DoS attack, launched by an attacker whose aim is to make an access point (AP) unavailable to legitimate users. One of the most common DoS attacks on 802.11 based networks is to deplete the resources of the AP. A serious situation like this can occur when the AP receives a burst of connection requests. Their paper addressed this common DoS attack and proposed a lightweight puzzle, based on pattern matching. Using a pattern-matching technique, this model adequately resisted resource-depletion attacks in terms of both puzzle generation and solution verification. Using a sensible series of contextual comparisons, the outcomes were modelled by a simulator, and the security definition and proofs are verified, among other results.

In the paper entitled "Weight Optimization in Recurrent Neural Networks with Hybrid Metaheuristic Cuckoo Search Techniques for Data Classification," N. M. Nawi et al. investigated recurrent neural network (RNN). This network can be educated with gradient descent backpropagation. However, traditional training algorithms had some drawbacks such as slow speed of convergence being not definite to find the global minimum of the error function since gradient descent may get stuck in local minima. As a solution, nature inspired metaheuristic algorithms provide derivative-free solution to optimize complex problems. This paper proposed a new metaheuristic search algorithm called Cuckoo Search (CS) based on Cuckoo bird's behavior to train Elman recurrent network (ERN) and backpropagation Elman recurrent network (BPERN) in achieving fast convergence rate and to avoid local minima problem. The proposed CSERN and CSBPERN algorithms were compared with artificial bee colony using $\mathrm{BP}$ algorithm and other hybrid variants algorithms. Specifically, some selected benchmark classification problems were used. The simulation results showed that the computational efficiency of ERN and BPERN training process was highly enhanced when coupled with the proposed hybrid method.

In the paper entitled "Stereo Matching Based on Immune Neural Network in Abdomen Reconstruction," H. Liu et al. suggested stereo feature matching is a technique that finds an optimal match in two images from the same entity in the three-dimensional world. The stereo correspondence problem is formulated as an optimization task where an energy function, which represents the constraints on the solution, is to be minimized. They proposed a novel intelligent biological network (Bio-Net), which involves the human B-T cells immune system into neural network, in order to learn the robust relationship between the input feature points and the output matched points. In the experiments, the abdomen reconstructions for different-shape mannequins were performed by means of the proposed method. The final results were compared and analyzed, which demonstrate that the proposed approach greatly outperforms the single neural network and the conventional matching algorithm in precise. Particularly, with respect to time cost and efficiency, the proposed method exhibits its significant promising potential for improvement. Hence, it is entirely considered as an effective and feasible alternative option for stereo matching.

In the paper entitled "Neural Network-Based FaultTolerant Control of Underactuated Surface Vessels," B. S. Park addressed the problem of trajectory tracking of underactuated surface vessels (USVs) in the presence of thruster failure. Multilayer neural networks (MNNs) were employed to estimate the unknown model parameters and external disturbances. To design a fault-tolerant controller without a fault detection scheme, they used the Nussbaum gain technique. They also introduced an additional control to resolve the difficulty arising from having fewer inputs than degrees of freedom. Further, an approach angle was proposed to track both straight 
and curved path. Stability analysis and simulations were performed to demonstrate the effectiveness of the proposed scheme.

In the paper entitled "Dynamic Track Management in MHT for Pedestrian Tracking Using Laser Range Finder," A. H. A. Rahman et al. proposed a multilevel clustering of laser range finder (LRF) data to improve the accuracy of a tracking system by adding another clustering level after the feature extraction process. A dynamic track management (DTM) was introduced in multiple hypothesis tracking (MHT) with multiple motion models to perform a track creation, association, and deletion. The experimental results from real time implementation proved that the proposed multiclustering is capable of producing a better performance with less computational complexity for a track management process. The proposed dynamic track management is able to solve the tracking problem with lower computation time when dealing with occlusion, crossed track, and track deletion.

In the paper entitled "A Study on Many-Objective Optimization Using the Kriging-Surrogate-Based Evolutionary Algorithm Maximizing Expected Hypervolume Improvement," C. Luo et al. investigated and compared the manyobjective optimization performance of the Kriging-surrogate-based evolutionary algorithm (EA), which maximizes expected hypervolume improvement (EHVI) for updating the Kriging model, with those using expected improvement (EI) and estimation (EST) updating criteria. Numerical experiments were conducted in 3- to 15-objective DTLZ17 problems. An exact hypervolume calculating algorithm was used for the problems with less than six objectives. On the other hand, an approximate hypervolume calculating algorithm based on Monte Carlo sampling was adopted for the problems with more objectives. The results indicate that, in the nonconstrained case, EHVI is a highly competitive updating criterion for the Kriging model and EA based manyobjective optimization, especially when the test problem is complex and the number of objectives or design variables is large.

In the paper entitled "System Optimization for Temporal Correlated Cognitive Radar with EBPSK-Based MCPC Signal," P. Chen and L. Wu proposed a novel radar working scheme to consider both target detection and estimation. At the detection stage, the generalized likelihood ratio test (GLRT) threshold was deduced, and the GLRT detection probability was given. At the estimation stage, an approach based on Kalman filtering (KF) was proposed to estimate target scattering coefficients (TSC), and the estimation performance was improved significantly by exploiting the TSC temporal correlation. Additionally, the optimal waveform was obtained to minimize the mean squared error (MSE) of KF estimation. For the practical consideration, iteration algorithms were proposed to optimize the EBPSKbased multicarrier phase-coded (MCPC) signal in terms of power allocation and coding matrix. Simulation results demonstrated that the KF estimation approach can improve the estimation performance by $25 \%$ compared with maximum a posteriori probability (MAP) method, and the KF estimation performance can be further improved by $90 \%$ by optimizing the transmitted waveform spectrum.
Moreover, by optimizing the power allocation and coding matrix of the EBPSK-based MCPC signal, the KF estimation performances were, respectively, improved by $7 \%$ and $8 \%$.

In the paper entitled "Color Image Encryption Algorithm Based on TD-ERCS System and Wavelet Neural Network," K. Zhang and J. Fang proposed a new image encryption algorithm based on TD-ERCS system and wavelet neural network, in order to solve the security problem of transmission image across public networks. According to the permutation process and the binary XOR operation from the chaotic series by producing TD-ERCS system and wavelet neural network, it can achieve image encryption. This encryption algorithm was a reversible algorithm, and it can achieve original image in the rule inverse process of encryption algorithm. Finally, through computer simulation, the experiment results showed that the new chaotic encryption algorithm based on TDERCS system and wavelet neural network is valid and has higher security.

In the paper entitled "Fault Diagnosis of Supervision and Homogenization Distance Based on Local Linear Embedding Algorithm," G. Wang et al. developed an improved local linear embedding algorithm of homogenization distance (HLLE), in view of the problems of uneven distribution of reality fault samples and dimension reduction effect of locally linear embedding (LLE) algorithm, which is easily affected by neighboring points. The method made the overall distribution of sample points tend to be homogenization and reduces the influence of neighboring points using homogenization distance instead of the traditional Euclidean distance. It was helpful to choose effective neighboring points to construct weight matrix for dimension reduction. Because the fault recognition performance improvement of HLLE was limited and unstable, the paper further proposed a new local linear embedding algorithm of supervision and homogenization distance (SHLLE) by adding the supervised learning mechanism. On the basis of homogenization distance, supervised learning increased the category information of sample points, so that the same category of sample points will be gathered and the heterogeneous category of sample points will be scattered. It effectively improved the performance of fault diagnosis and maintains stability at the same time. A comparison of the methods mentioned above was made by simulation experiment with rotor system fault diagnosis, and the results showed that SHLLE algorithm has superior fault recognition performance.

In the paper entitled "A New Asymptotic Notation: Weak Theta," A.-H. Mogos et al. defined a new asymptotic notation, called "Weak Theta," that used the comparison of various complexity functions with two given complexity functions. Weak Theta notation was especially useful in characterizing complexity functions whose behavior was hard to be approximated using a single complexity function. In addition, in order to highlight the main particularities of Weak Theta, they proposed and proved several theoretical results: properties of Weak Theta, criteria for comparing two complexity functions, and properties of a new set of complexity functions based on Weak Theta. Furthermore, to illustrate the usefulness of this notation, 
the authors discussed an application of Weak Theta in artificial intelligence.

In the paper entitled "Locating High-Impedance Fault Section in Electric Power Systems Using Wavelet Transform, $k$-Means, Genetic Algorithms, and Support Vector Machine," Y.-Y. Hong and W.-S. Huang presented a new method for locating the line (feeder) section of the high-impedance fault (HIF) with the help of limited measurements in electric power systems. The discrete wavelet transform was used to extract the features of transients caused by HIFs. A modified $k$-means algorithm associated with genetic algorithms was then utilized to determine the placement of measurement facilities. The signal energies attained by wavelet coefficients served as inputs to the support vector machine for locating the HIF line section. The simulation results obtained from an 18-busbar distribution system showed the applicability of the proposed method.

In the paper entitled "Recognition of Mixture Control Chart Pattern Using Multiclass Support Vector Machine and Genetic Algorithm Based on Statistical and Shape Features," M. Zhang and W. Cheng introduce an intelligent hybrid model for recognizing the mixture control chart patterns (CCPs) that included three main aspects: feature extraction, classifier, and parameters optimization. In the feature extraction, statistical and shape features of observation data were used in the data input to get the effective data for the classifier. A multiclass support vector machine (MSVM) was applied for recognizing the mixture CCPs. Finally, genetic algorithm (GA) was utilized to optimize the MSVM classifier by searching the best values of the parameters of MSVM and kernel function. The performance of the hybrid approach was evaluated by simulation experiments, and simulation results demonstrated that the proposed approach was able to effectively recognize mixture CCPs.

In the paper entitled "A Fault-Tolerant Filtering Algorithm for SINS/DVL/MCP Integrated Navigation System," $\mathrm{X}$. Xu et al. proposed a fault-tolerant adaptive Kalman filter (FTAKF) algorithm for the integrated navigation system composed of a strapdown inertial navigation system (SINS), a Doppler velocity log (DVL), and a magnetic compass (MCP). The evolutionary artificial neural networks (EANN) were used in self-learning and training of the intelligent data fusion algorithm. The proposed algorithm can significantly outperform the traditional KF in providing estimation continuously with higher accuracy and smoothing the KF outputs when observation data were inaccurate or unavailable for a short period. The experiments of the prototype verified the effectiveness of the proposed method.

In the paper entitled "Multiagent Cooperative Learning Strategies for Pursuit-Evasion Games," J. Y. Kuo et al. examined the pursuit-evasion problem for coordinating multiple robotic pursuers to locate and track a nonadversarial mobile evader in a dynamic environment. Two kinds of pursuit strategies were proposed, one for agents that cooperate with each other and the other for agents that operate independently. This work further employed the probabilistic theory to analyze the uncertain state information about the pursuers and the evaders and uses case-based reasoning to equip agents with memories and learning abilities. According to the concepts of assimilation and accommodation, both positive-angle and bevel-angle strategies were developed to assist agents in adapting to their environment effectively. The case study analysis used the recursive porous agent simulation toolkit (REPAST) to implement a multiagent system and demonstrated superior performance of the proposed approaches to the pursuit-evasion game.

In the paper entitled "User Adapted Motor-Imaginary Brain-Computer Interface by means of EEG Channel Selection Based on Estimation of Distributed Algorithms," A. Astigarraga et al. presented a personalized interface design method, for electroencephalogram (EEG) based BrainComputer Interfaces (BCIs), based on channel selection. They described a novel two-step method in which firstly a computationally inexpensive greedy algorithm found an adequate search range; and then, an estimation of distribution algorithm (EDA) was applied in the reduced range to obtain the optimal channel subset. The use of the EDA algorithm allowed us to select the most interacting channels subset, removing the irrelevant and noisy ones, thus selecting the most discriminative subset of channels for each user improving accuracy. The method was tested on the IIIa dataset from the BCI competition III. Experimental results showed that the resulting channel subset was consistent with motor imaginary related neurophysiological principles and, on the other hand, optimized performance reducing the number of channels.

In the paper entitled "KD-ACP: A Software Framework for Social Computing in Emergency Management," B. Chen et al. addressed the application of a computational theory and related techniques for studying emergency management in social computing. They proposed a novel software framework called KD-ACP. The framework provided a systematic and automatic platform for scientists to study the emergency management problems in three aspects: modelling the society in emergency scenario as the artificial society; investigating the emergency management problems by the repeat computational experiments; parallel execution between artificial society and the actual society managed by the decisions from computational experiments. The software framework was composed of a series of tools. These tools were categorized into three parts corresponding to " $\mathrm{A}$," "C," and "P," respectively. Using H1N1 epidemic in Beijing city as the case study, the modelling and data generating of Beijing city, experiments with settings of H1N1, and intervention measures and parallel execution by situation tool were implemented by KD-ACP. The results output by the software framework showed that the emergency response decisions can be tested to find a more optimal one through the computational experiments. In the end, the advantages of the KD-ACP and the future work were summarized in the conclusion.

\section{Acknowledgments}

We would like to show our appreciation to all authors for their contributions and the reviewers for their effort providing 
valuable and prompt comments. We hope that this special issue offers a comprehensive and timely view of the advances in artificial intelligence and its applications, and we expect that it will offer stimulation for further research.

Yudong Zhang

Saeed Balochian

Praveen Agarwal

Vishal Bhatnagar

Orwa Jaber Housheya 


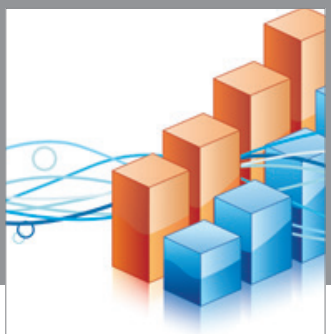

Advances in

Operations Research

vatem alat4

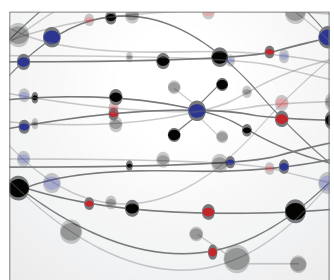

\section{The Scientific} World Journal
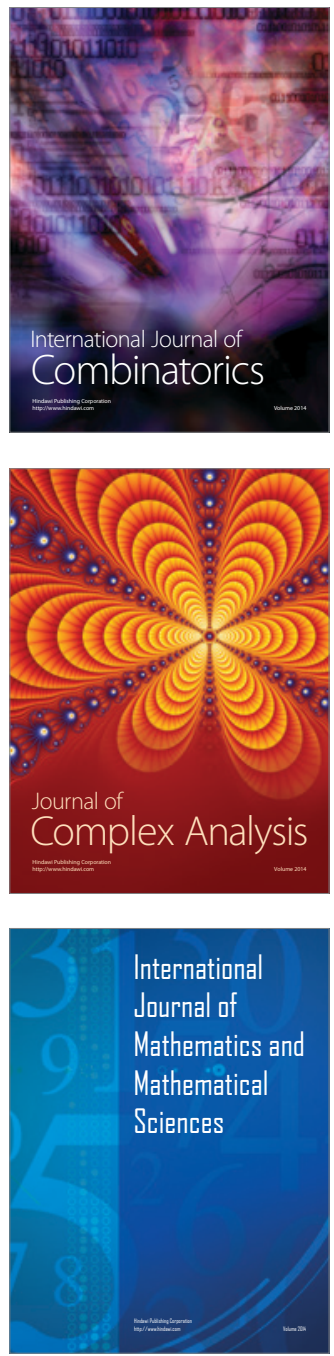
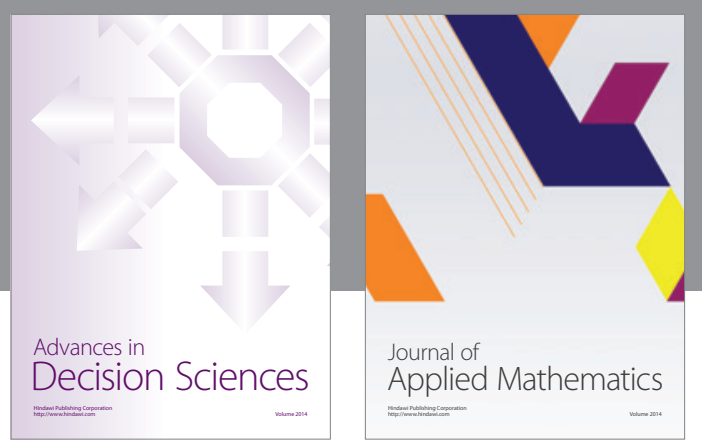

Algebra

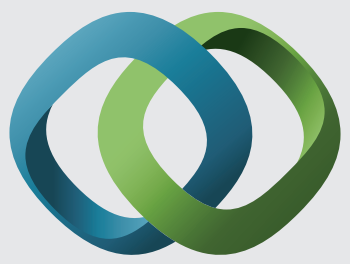

\section{Hindawi}

Submit your manuscripts at

http://www.hindawi.com
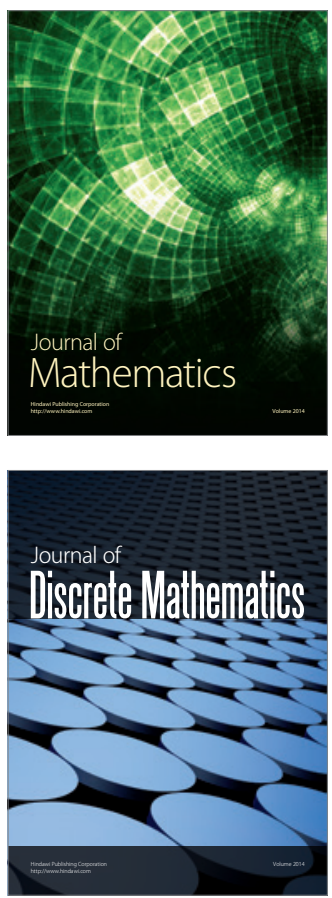

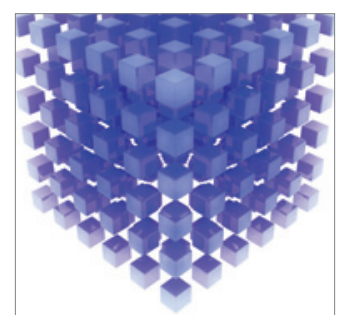

Mathematical Problems in Engineering
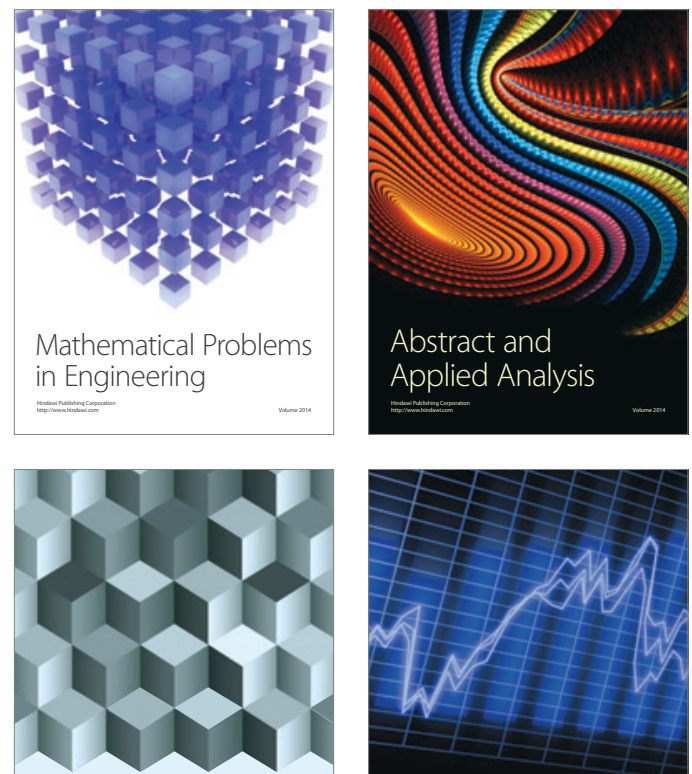

Journal of

Function Spaces

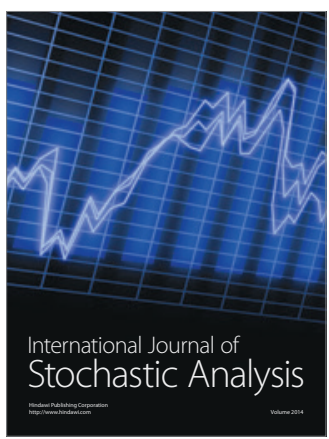

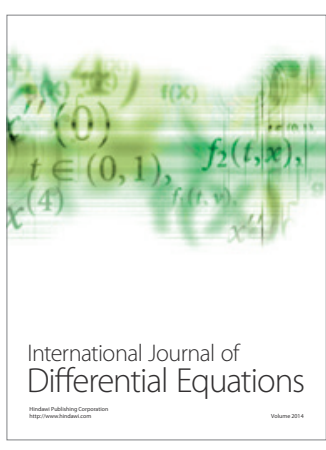
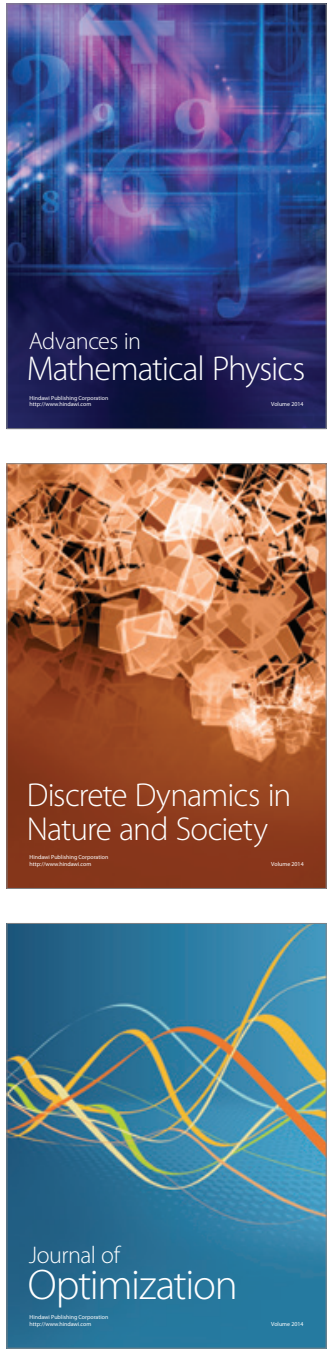